\title{
Takotsubo cardiomyopathy in a 12-year-old boy caused by acute brainstem bleeding - a case report
}

\author{
Clara Thomas $^{1 \wedge}$, Sarah M. Johler ${ }^{1}$, Matthias Hermann ${ }^{1}$, Marcus Fischer ${ }^{1}$, Jun Thorsteinsdottir ${ }^{2}$, \\ Christian Schichor ${ }^{2}$, Nikolaus A. Haas ${ }^{1}$ \\ ${ }^{1}$ Department for Pediatric Cardiology and Intensive Care, Medical Hospital of the University of Munich, Ludwig Maximilians University Munich, \\ Munich, Germany; ${ }^{2}$ Department for Neurosurgery, Medical Hospital of the University of Munich, Ludwig Maximilians University Munich, Munich, \\ Germany \\ Correspondence to: Clara Thomas, MD. Department for Pediatric Cardiology and Intensive Care, Medical Hospital of the University of Munich, \\ Ludwig Maximilians University Munich, Marchioninistr. 15, 81377 Munich, Germany. Email: clarathomas@web.de.
}

\begin{abstract}
Takotsubo cardiomyopathy is characterized by acute and reversible severe left ventricular dysfunction due to intensive emotional or physical stress followed by catecholamine excess. Traditionally it is most common in postmenopausal women, whereas only few cases have been described in childhood. In our case a previously well 12-year-old boy presented with severe cardiogenic shock due to dramatically impaired left ventricular function requiring significant inotropic support and invasive mechanical ventilation. Interestingly, cardiac catheterization, myocardial tissue histology and biochemical laboratory tests did not yield a definitive diagnosis. As his cardiac function improved gradually within several days and deep sedation could be weaned, he was then found to suffer from hemiparesis and absence of protective airway reflexes on neurological examination during the weaning process. Subsequent brain imaging studies revealed a brainstem bleeding due to a fistulous arteriovenous malformation (AVM) appearing to be only a few days old. After endovascular coiling and subsequent microsurgical resection of the malformation, he recovered completely. Our present case demonstrated, that brainstem bleeding could precipitate Takotsubo cardiomyopathy manifesting hemodynamic collapse. Severe ventricular impairment has been described in many adults with subarachnoid hemorrhage; however, this condition is extremely rare among children. When severe cardiogenic shock is diagnosed, precipitating factors such as intracranial processes should be ruled out on a regular basis.
\end{abstract}

Keywords: Takotsubo cardiomyopathy; stress-cardiomyopathy; child; intracranial hemorrhage; case report

Submitted Apr 21, 2021. Accepted for publication Sep 29, 2021.

doi: $10.21037 /$ tp-21-181

View this article at: https://dx.doi.org/10.21037/tp-21-181

\section{Introduction}

\section{Background: Takotsubo cardiomyopathy}

Since its' first description in Japan in the early 1990s, the awareness of Takotsubo cardiomyopathy has risen in the past years $(1,2)$. Inspired by its typical configuration with systolic apical ballooning of the left ventricle (LV), it was named after the traditional Japanese octopus trap (3). It is characterized as acute and severe left ventricular dysfunction mimicking acute coronary syndrome in clinical symptoms until cardiogenic shock, elevation of cardiac enzymes and electrocardiographic (ECG) alterations (4). Lacking the underlying coronary artery stenosis, it is rather based on intensive emotional or physical stress causing catecholamine

^ ORCID: 0000-0003-3433-8774. 
Table 1 Vital parameters and clinical status of a 12-year-old boy presenting to the ED in severe cardiogenic shock later diagnosed with Takotsubo cardiomyopathy caused by acute brainstem bleeding

\begin{tabular}{ll}
\hline Vital parameters and clinical status & Value \\
\hline Blood pressure $(\mathrm{mmHg})$ & $105 / 53$ \\
Heart rate $(\mathrm{bpm})$ & 139 \\
Oxygen saturation (\%) & $55\left(\right.$ with $\left.\mathrm{FiO}_{2} 1.0\right)$ \\
Cor & Rhythmic, systolic murmur, weak peripheral pulses palpable \\
Pulmo & Symmetrical ventilation, ubiquitous wheezing, crackles \\
Abdomen & No resistances, no pressure pain, liver and spleen not enlarged, normal bowel sounds \\
Throat & Uremarkable \\
Skin & Pale, limbs cool, peripheral capillary refill 5 seconds, no signs of chronic cyanosis, no exanthema \\
Neuro & Somnolence, low muscle tone
\end{tabular}

$\mathrm{ER}$, emergency department; $\mathrm{FiO}_{2}$, fraction of inspired oxygen.

excess $(4,5)$. As left ventricular dysfunction is typically reversible in this condition, patients with Takotsubo cardiomyopathy generally have a favorable prognosis (4).

\section{Focus: stress-induced cardiomyopathy in children}

While most common among adults, especially elderly women (2), stress-induced cardiomyopathy has very rarely been described in children, presenting similar clinical symptoms and diagnostic findings as adults $(3,6,7)$. In childhood however, it is often initially mistaken for much more common diagnoses like dilated cardiomyopathy or myocarditis or even rare differential diagnoses like previous untreated Kawasaki's disease, undiagnosed congenital anomalous coronary artery origin or permanent junctional reentry tachycardia (3).

We present the following case in accordance with the CARE reporting checklist (available at https://dx.doi. org/10.21037/tp-21-181).

\section{Case presentation}

\section{Ethical statement}

All procedures performed in studies involving human participants were in accordance with the ethical standards of the institutional and/or national research committee(s) and with the Helsinki Declaration (as revised in 2013). Written informed consent was obtained from the patient's parent or legal guardian for publication of this case report and accompanying images. A copy or the written informed consent is available for review by the editorial office of this journal.

\section{Medical bistory and clinical presentation}

The 12-year-old boy presented in our emergency department (ED) complaining about an afebrile illness with pain in the jaw, mouth and throat since the past night. Selfmedication with paracetamol had brought no improvement, instead he had suddenly developed muscular hypotonia, oral leaking of bloody, foamy saliva and pulmonary wheezing. He had no pre-existing medical conditions or allergies, no regular medication and no family history for cardiovascular diseases.

During evaluation in the ED drastically deteriorating cardiovascular parameters (see Table 1) and decreasing saturation of $50 \%$ under oxygen therapy resulted in a collapse to somnolence.

Emergency echocardiography showed massive acute left ventricular functional restriction (see Figure 1 and Table 2).

Catecholamine therapy (Adrenaline and Milrinone) was initiated and after intubation the boy was transferred to pediatric intensive care for further evaluation and potential extracorporeal life support (ECLS) therapy.

\section{Diagnostic pathway and initial therapy}

On admission, the patient was hypotensive despite high doses of catecholamines. Initial ECG showed tachycardic sinus rhythm (140 bpm) with ST-depressions in II, III, augmented voltage foot (aVF) and elevations in V1-4 (see 

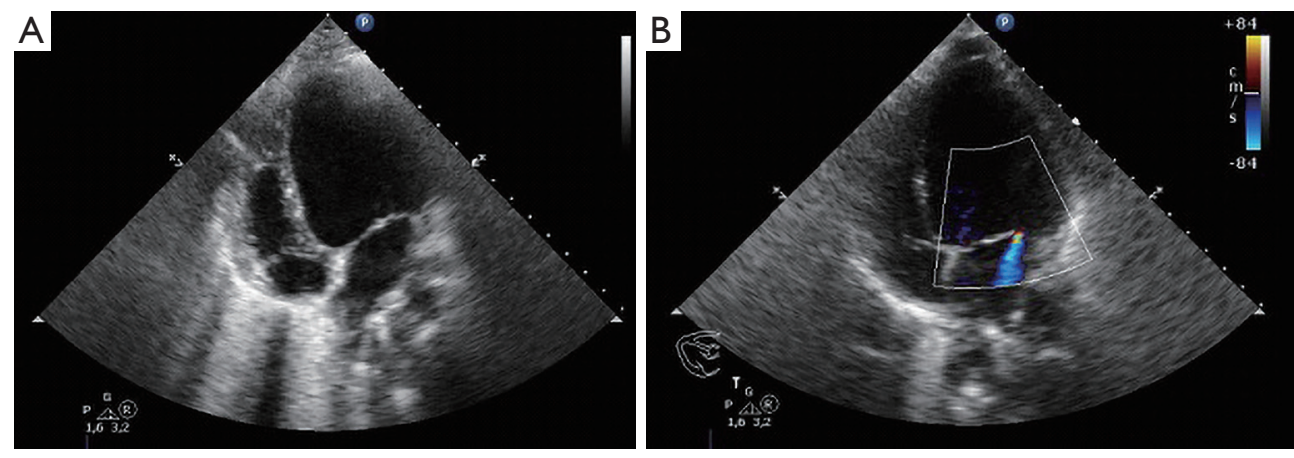

Figure 1 Emergency echocardiography of a 12-year-old boy with Takotsubo cardiomyopathy caused by acute brainstem bleeding showing (A) $\mathrm{LV}$ dilatation and (B) MI III'. LV, left ventricle; MI, mitral insufficiency.

Figure 2), chest X-ray revealed pulmonary oedema (see Figure 3). Blood gases showed lactate acidosis (lactate max. $8 \mathrm{mmol} / \mathrm{L}, \mathrm{pH} \min .7 .22$ ) and cardiac enzymes were highly increased (see Figure 2).

Left ventricular function and cardiac output was just sufficient to refrain from ECLS therapy. Suspecting acute viral myocarditis and to rule out coronary pathologies, cardiac catheterization including myocardial biopsies was performed. It showed severely impaired cardiac function lacking any coronary anomalies while the biopsies revealed no signs of myocarditis (see Table 2).

Within the subsequent days, the boy's cardiac function steadily stabilized under medical therapy (Adrenaline max. $0.2 \mu \mathrm{g} / \mathrm{kg} / \mathrm{min}$, Noradrenaline max. $0.1 \mu \mathrm{g} / \mathrm{kg} / \mathrm{min}$, Milrinone max. $1 \mu \mathrm{g} / \mathrm{kg} / \mathrm{min}$, one cycle of Levosimendan), catecholamine therapy could be reduced and terminated after a few days.

Blood gases normalized after 1 day and cardiac enzymes decreased to normal after 1 week [Troponin $\mathrm{T}$ (hs) max. $1.46 \mathrm{ng} / \mathrm{mL} 1$ day after admission, NT-pro brain natriuretic peptide (NT-proBNP) max. 26,833 pg/mL 3 days after admission, see Figure 2]. As all other investigations were negative (see Table 2), the underlying diagnosis remained unclear.

\section{Clinical plot twist and outcome}

During the weaning process and while preparing extubation with reduction of analgosedation the boy presented with the absence of protective gagging, coughing and swallowing reflexes (no reaction to endotracheal and oral suction) and right hemiparesis (right-sided motor weakness when compared to the left side, like when pressing doctors hand or pushing with feet).
Subsequent cerebral MRI revealed a hematoma in the dorsolateral caudal medulla oblongata with intraventricular blood accumulation that was judged only few days old and sufficiently explained the lack of brainstem reflexes as well as the motor weakness (see Figure $4 A$ ). A perimedullary fistulous arteriovenous malformation (AVM) was identified as the cause of the bleeding (see Figure $4 B$ ). After partial endovascular coiling and subsequent surgical occlusion of the perimedullary AVM (see Figure 5) the patient was successfully extubated, but only gradually improved neurologically. He was finally transferred to a neurological rehabilitation center with severe right hemiparesis but completely recovered cardiac function. Follow-up examinations after 3 months showed no remains of the AVM and normal cardiac function.

Takotsubo cardiomyopathy was diagnosed retrospectively as the patient met all four of the suggested Mayo Clinic diagnostic criteria for this type of cardiomyopathy (8).

\section{Discussion}

Our patient presented in severe cardiogenic shock with drastically impaired left ventricular function. Initially suspecting dilated cardiomyopathy or myocarditis, specific cardiologic diagnostics did not show pathological findings yielding to a diagnosis. When additional neurological symptoms got obvious during the weaning process, a brainstem bleeding due to a fistulous AVM was identified. Thus only in retrospect, the neurological condition was interpreted as precipitating cause of the acute ventricular impairment yielding to the diagnosis of Takotsubo cardiomyopathy.

Takotsubo cardiomyopathy is extremely rare in children and like in our case often initially mistaken for myocarditis 
Table 2 Diagnostic investigations and results during in-hospital stay of a 12-year-old boy in severe cardiogenic shock later diagnosed with Takotsubo cardiomyopathy caused by acute brainstem bleeding

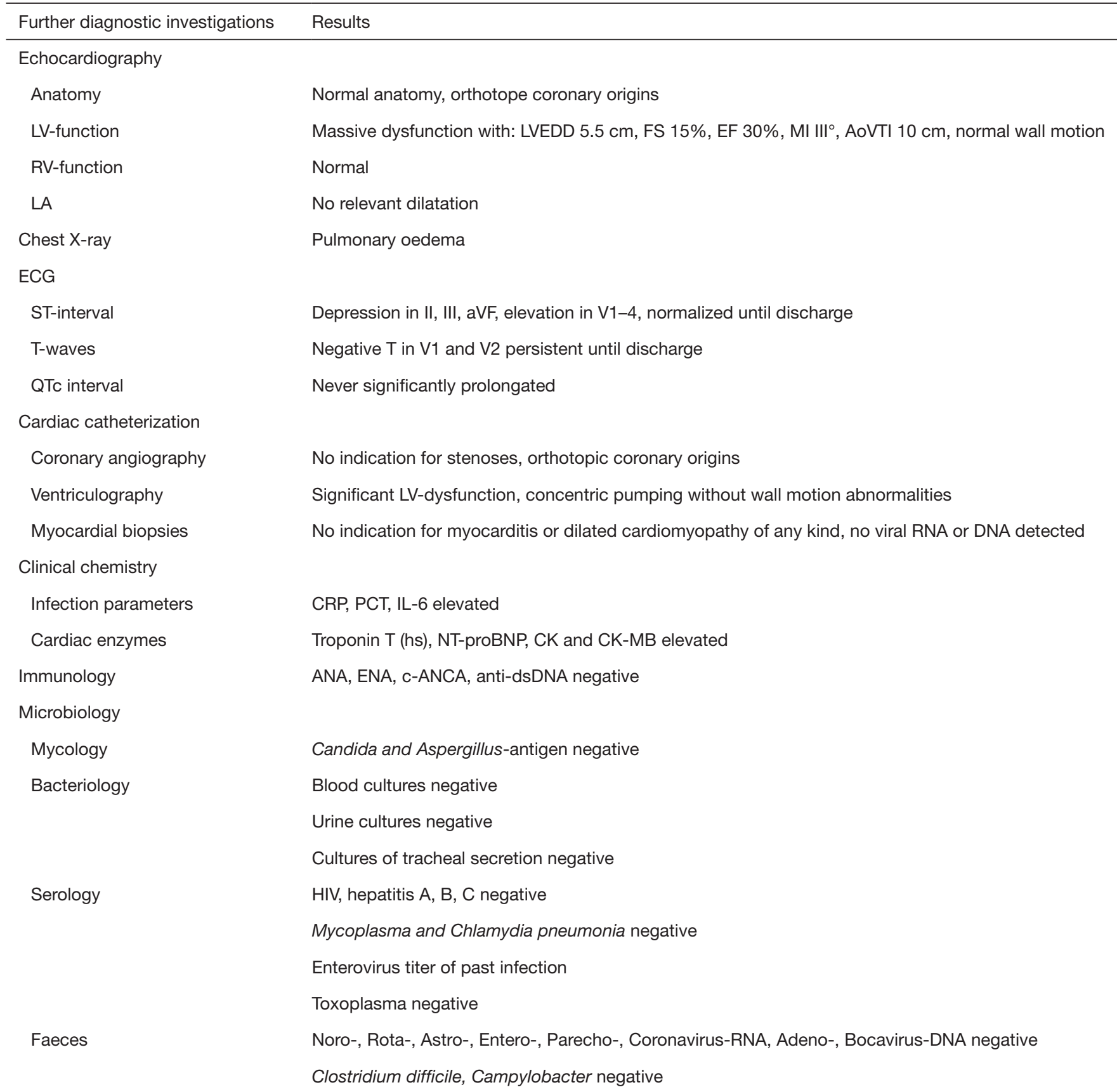

LV, left ventricle; LVEDD, left ventricular end diastolic diameter; FS, fractional shortening; EF, ejection fraction; MI, mitral insufficiency; AoVTI, aortal velocity time integral; RV, right ventricle; LA, left atrium; ECG, electrocardiogram; aVF, augmented voltage foot; CRP, C-reactive protein; PCT, procalcitonin; IL-6, interleukin-6; NT-proBNP, NT-pro brain natriuretic peptide; CK, creatine kinase; CK-MB, muscle-braintype creatine kinase; ANA, antinuclear antibodies; ENA, extractable nuclear antigen; c-ANCA, antineutrophil cytoplasmic antibodies; antidsDNA, anti-double stranded DNA antibodies; HIV, human immunodeficiency virus. 


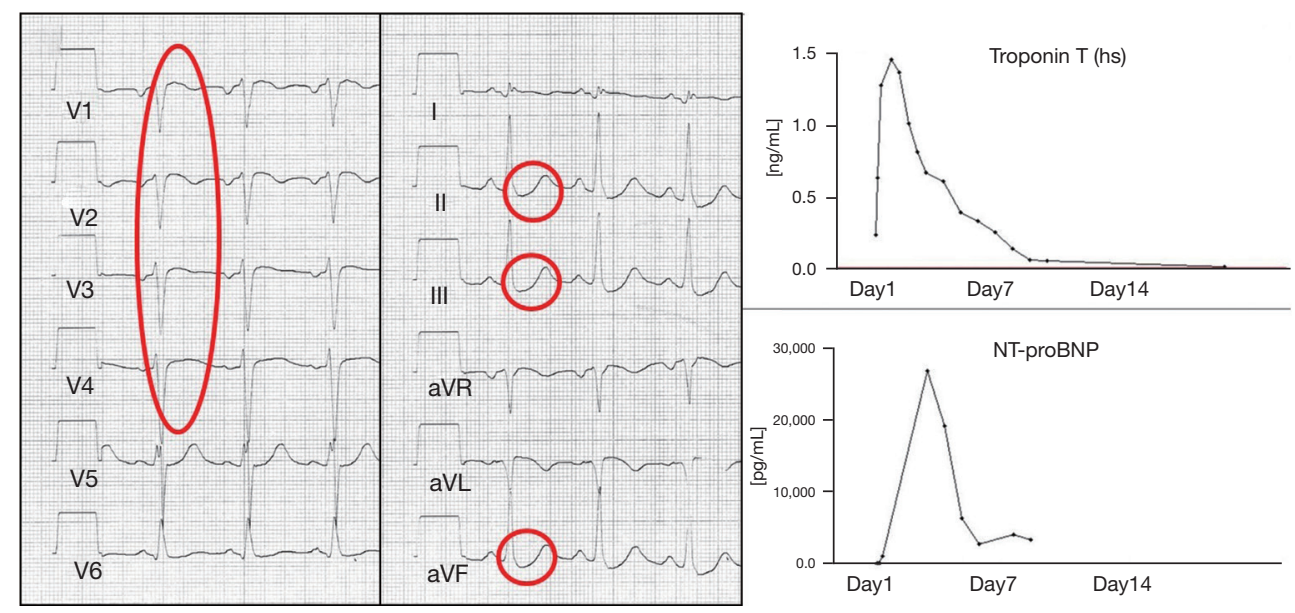

Figure 2 Diagnostic findings in a 12-year-old boy with Takotsubo cardiomyopathy caused by acute brainstem bleeding showing (A) ECG with tachycardic sinus rhythm (140 bpm) with ST-depressions in II, III, aVF and elevations in V1-4 (red circles) and (B) highly elevated cardiac enzymes which nearly normalized after 1 week. ECG, electrocardiogram; aVF, augmented voltage foot; NT-proBNP, NT-pro brain natriuretic peptide.

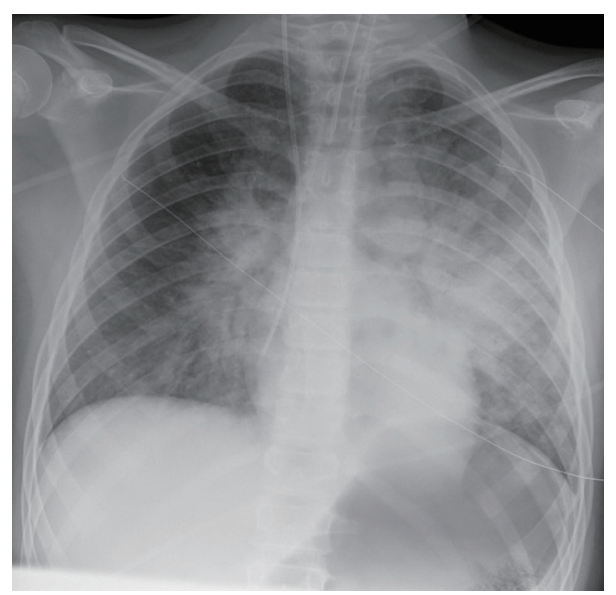

Figure 3 Initial chest X-ray of a 12-year-old boy with Takotsubo cardiomyopathy caused by acute brainstem bleeding after intubation and placement of a central line in the ED, showing pulmonary oedema. ED, emergency department.

or dilated cardiomyopathy (3). Previously described triggering conditions range from emotional stressors (9-11) to physical conditions (12-15). While the exact pathophysiologic mechanisms of Takotsubo cardiomyopathy remain unclear, the most distributed hypothesis implicates myocardial stunning. Sympathetic dysregulation and subsequent catecholamine excess supposedly cause direct toxic cardiomyocyte damage as well as coronary spasms in face of higher cardiac workload $(5,16,17)$. Neurological and neurosurgical disorders as causes of Takotsubo cardiomyopathy seem less common in children than in adult patients (6) and include intracerebral hemorrhage, in some cases due to AVMs and fistulas (7,18-22). Like in our case, especially lesions affecting the medulla, home to important regulators of the autonomic function, can furthermore cause dysregulation of blood pressure and heart rate due to baroreflex failure resulting in cardiogenic shock $(17,23,24)$.

While the prognosis of Takotsubo cardiomyopathy is favorable in general (4), fatal outcomes have also been reported $(10,19)$.

\section{Conclusions}

* Acute myocarditis and dilated cardiomyopathy are the most common diagnoses in severe pediatric heart failure;

* Takotsubo cardiomyopathy as a rare but possible cause for secondary acute left ventricular dysfunction and cardiogenic shock should not be disregarded;

* Precipitating etiological factors such as intracranial processes should be ruled out on a regular basis. 

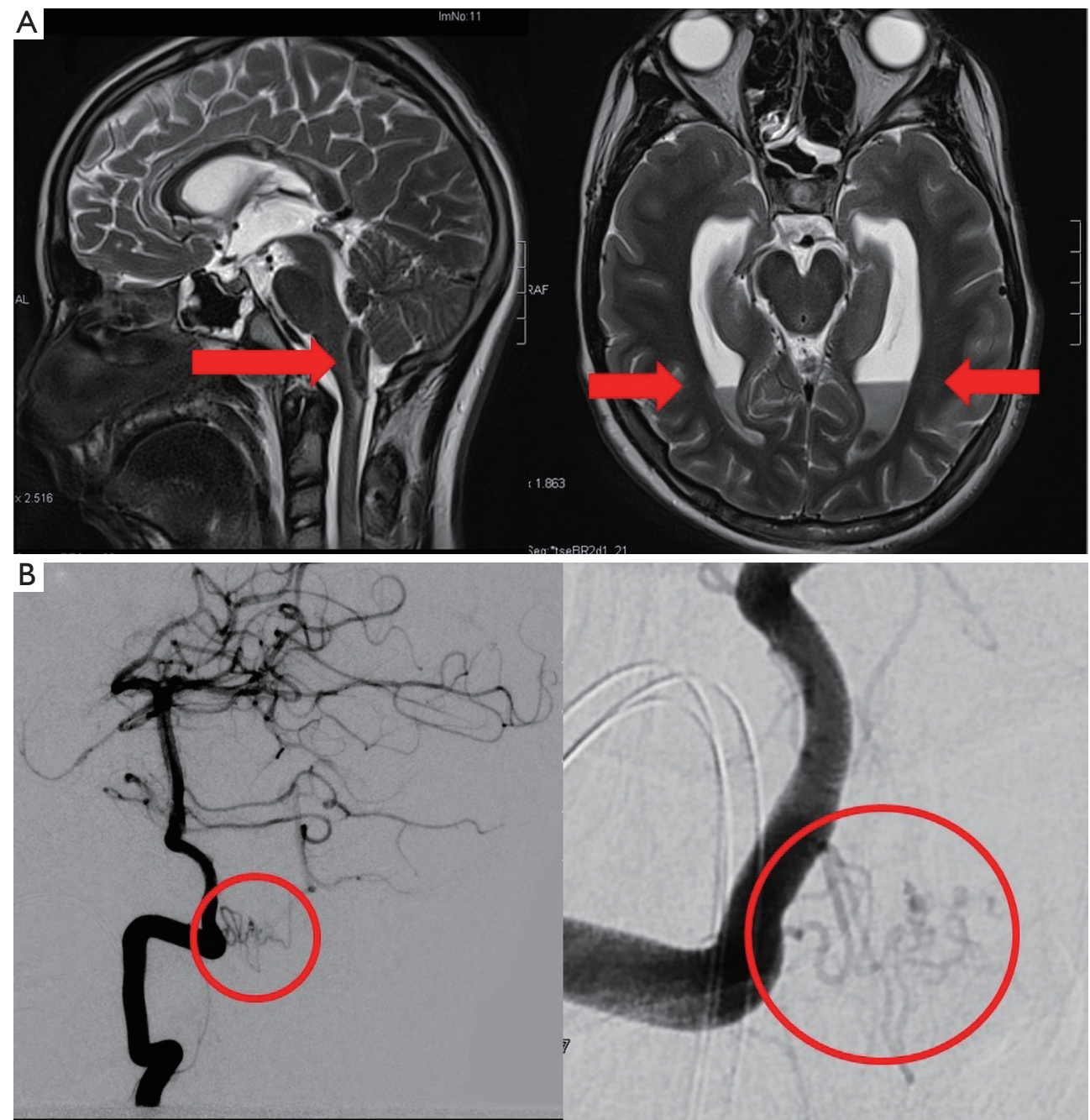

Figure 4 Cerebral imaging in a 12-year-old boy with Takotsubo cardiomyopathy caused by (A) hematoma close to the dorsocaudal medulla oblongata (red arrow left image) with intraventricular blood accumulation in the lateral ventricles (red arrows right image) based on (B) a fistulous AVM (red circles) adjacent to the medulla oblongata. AVM, arteriovenous malformation. 

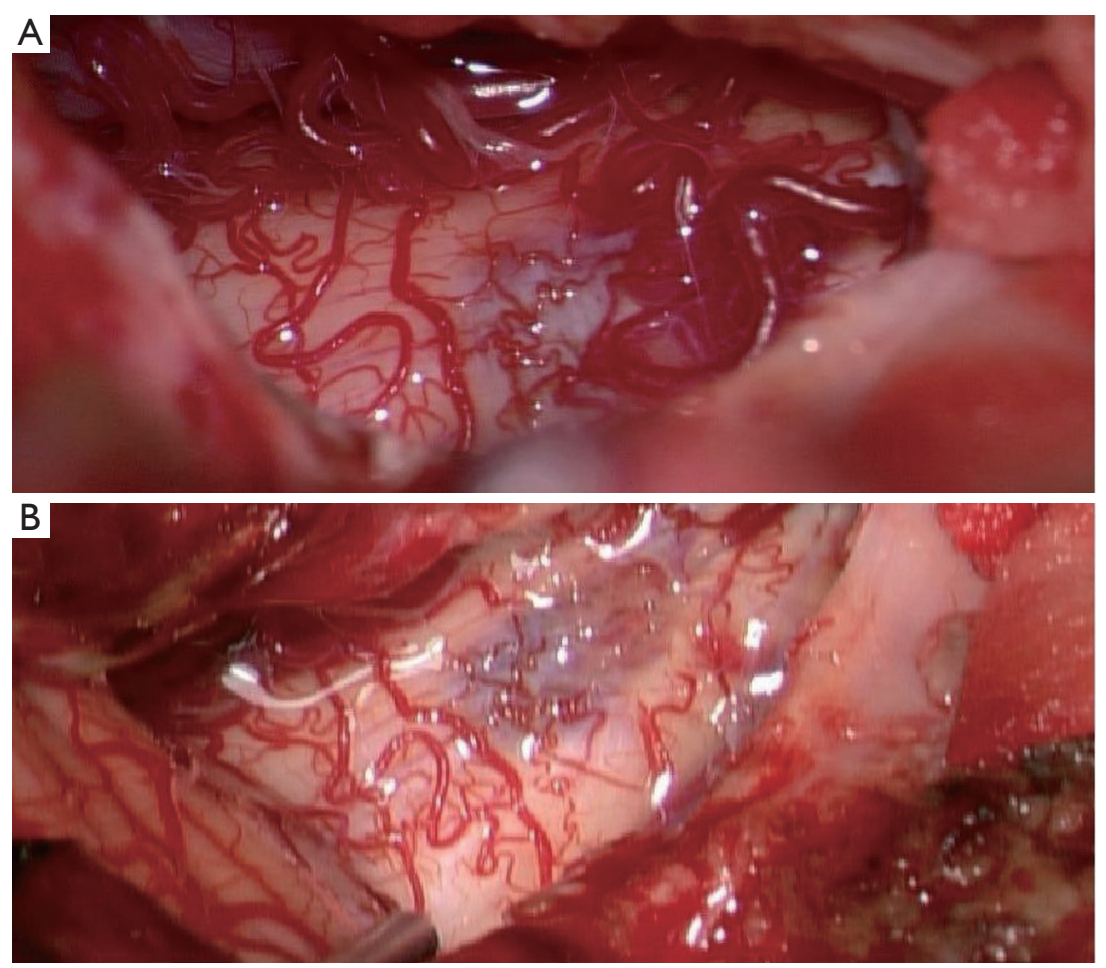

Figure 5 Intraoperative images of a 12-year-old boy with Takotsubo cardiomyopathy caused by acute brainstem bleeding showing (A) the fistulous AVM on the dorsal medulla oblongata and (B) the postoperative status with complete resection of the fistulous AVM. AVM, arteriovenous malformation.

\section{Acknowledgments}

Parts of this case have been previously presented at the annual conference of the Deutsche Gesellschaft für Pädiatrische Kardiologie, Wiesbaden, Germany 2020. The authors thank their patient and his mother for their permission to publish this article.

Funding: None.

\section{Footnote}

Reporting Checklist: The authors have completed the CARE reporting checklist. Available at https://dx.doi. org/10.21037/tp-21-181

Peer Review File: Available at https://dx.doi.org/10.21037/ tp-21-181

Conflicts of Interest: All authors have completed the ICMJE uniform disclosure form (available at https://dx.doi. org/10.21037/tp-21-181). The authors have no conflicts of interest to declare.
Ethical Statement: The authors are accountable for all aspects of the work in ensuring that questions related to the accuracy or integrity of any part of the work are appropriately investigated and resolved. All procedures performed in studies involving human participants were in accordance with the ethical standards of the institutional and/or national research committee(s) and with the Helsinki Declaration (as revised in 2013). Written informed consent was obtained from the patient's parent or legal guardian for publication of this case report and accompanying images. A copy or the written informed consent is available for review by the editorial office of this journal.

Open Access Statement: This is an Open Access article distributed in accordance with the Creative Commons Attribution-NonCommercial-NoDerivs 4.0 International License (CC BY-NC-ND 4.0), which permits the noncommercial replication and distribution of the article with the strict proviso that no changes or edits are made and the original work is properly cited (including links to both the formal publication through the relevant DOI and the license). 
See: https://creativecommons.org/licenses/by-nc-nd/4.0/.

\section{References}

1. Dote K, Sato H, Tateishi H, et al. Myocardial stunning due to simultaneous multivessel coronary spasms: a review of 5 cases. J Cardiol 1991;21:203-14.

2. Minhas AS, Hughey AB, Kolias TJ. Nationwide trends in reported incidence of takotsubo cardiomyopathy from 2006 to 2012. Am J Cardiol 2015;116:1128-31.

3. Hernandez LE. Takotsubo cardiomyopathy: how much do we know of this syndrome in children and young adults? Cardiol Young 2014;24:580-92.

4. Tsuchihashi K, Ueshima K, Uchida T, et al. Transient left ventricular apical ballooning without coronary artery stenosis: a novel heart syndrome mimicking acute myocardial infarction. Angina Pectoris-Myocardial Infarction Investigations in Japan. J Am Coll Cardiol 2001;38:11-8.

5. Pelliccia F, Kaski JC, Crea F, et al. Pathophysiology of Takotsubo syndrome. Circulation 2017;135:2426-41.

6. Finsterer J, Stöllberger C. Neurological and nonneurological triggers of Takotsubo syndrome in the pediatric population. Int J Cardiol 2015;179:345-7.

7. Urbinati A, Pellicori P, Guerra F, et al. Takotsubo syndrome in the paediatric population: a case report and a systematic review. J Cardiovasc Med (Hagerstown) 2017;18:262-7.

8. Madhavan M, Prasad A. Proposed Mayo Clinic criteria for the diagnosis of Tako-Tsubo cardiomyopathy and longterm prognosis. Herz 2010;35:240-3.

9. Biteker M, Duran NE, Civan HA, et al. Broken heart syndrome in a 17-year-old girl. Eur J Pediatr 2009;168:1273-5.

10. Zalewska-Adamiec $M$, Bachórzewska-Gajewska $\mathrm{H}$, Kralisz P, et al. Sudden cardiac arrest in the course of takotsubo syndrome in a 15-year-old girl. Postepy Kardiol Interwencyjnej 2018;14:318-9.

11. Bajolle F, Basquin A, Lucron H, et al. Acute ischemic cardiomyopathy after extreme emotional stress in a child. Congenit Heart Dis 2009;4:387-90.

12. Ohwada R, Hotta M, Kimura H, et al. Ampulla cardiomyopathy after hypoglycemia in three young female patients with anorexia nervosa. Intern Med 2005;44:228-33.

13. Hernandez LE, Martinez Y, Chan KC. Takotsubo cardiomyopathy: an unusual cardiomyopathy at an unusual age. Cardiol Young 2010;20:577-9.

14. Dalla Pozza R, Lehner A, Ulrich S, et al. Takotsubo cardiomyopathy complicating percutaneous pulmonary valve implantation in a child. World J Pediatr Congenit Heart Surg 2020;11:NP37-40.

15. Santoro F, Ieva R, Spennati G, et al. Tako-Tsubo cardiomyopathy in a teen girl with pheochromocytoma. Int J Cardiol 2012;160:e48-9.

16. Lee VH, Oh JK, Mulvagh SL, et al. Mechanisms in neurogenic stress cardiomyopathy after aneurysmal subarachnoid hemorrhage. Neurocrit Care 2006;5:243-9.

17. Gekka M, Yamaguchi S, Kazumata K, et al. Hemorrhagic onset of hemangioblastoma located in the dorsal medulla oblongata presenting with tako-tsubo cardiomyopathy and neurogenic pulmonary edema: a case report. Case Rep Neurol 2014;6:68-73.

18. Nakamori Y, Miyazawa N, Yoshitani K, et al. A pediatric case with Takotsubo cardiomyopathy and neurogenic pulmonary edema due to an epidural hemorrhage. J Neurosurg Anesthesiol 2018;30:279-80.

19. Haug KS, Baylen BG, Mink RB. Death from cardiac failure in a child with ruptured cerebral arteriovenous malformation. Pediatr Emerg Care 2009;25:342-4.

20. Wittekind SG, Yanay O, Johnson EM, et al. Two pediatric cases of variant neurogenic stress cardiomyopathy after intracranial hemorrhage. Pediatrics 2014;134:e1211-7.

21. Young YR, Lee CC, Sheu BF, et al. Neurogenic cardiopulmonary complications associated with spontaneous cerebellar hemorrhage. Neurocrit Care 2007;7:238-40.

22. Pearson TE, Frizzola MA, Priest MA, et al. Pediatric extracorporeal cardiopulmonary resuscitation patient with traumatic subarachnoid hemorrhage and Takotsubo syndrome. Air Med J 2018;37:64-6. Erratum in: Air Med J 2018;37:95.

23. Venkatraman A, Bajaj NS, Khawaja A, et al. Cardiogenic shock from atypical Takotsubo cardiomyopathy attributed to acute disseminated encephalomyelitis lesion involving the medulla. Clin Auton Res 2016;26:149-51.

24. Berganzo K, Ciordia R, Gómez-Esteban JC, et al. Takotsubo cardiomyopathy in a patient with bilateral lesions in the dorsal medulla. Clin Auton Res 2011;21:65-7.

Cite this article as: Thomas C, Johler SM, Hermann M, Fischer M, Thorsteinsdottir J, Schichor C, Haas NA. Takotsubo cardiomyopathy in a 12-year-old boy caused by acute brainstem bleeding—a case report. Transl Pediatr 2021;10(11):3110-3117. doi: $10.21037 /$ tp-21-181 